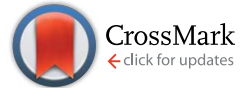

Cite this: J. Mater. Chem. A, 2016, 4, 6577

Received 22nd February 2016 Accepted 29th March 2016

DOI: 10.1039/c6ta01582d

www.rsc.org/MaterialsA

\title{
Direct photocatalytic hydrogen evolution from water splitting using nanostructures of hydrate organic small molecule as photocatalysts $\uparrow$
}

\begin{abstract}
Huihui Li, ${ }^{\text {ab }}$ Liulun Jie, ${ }^{\text {ab }}$ Jiannan Pan, ${ }^{\text {ab }}$ Longtian Kang ${ }^{\star a b}$ and Jiannian Yao*c
Organic small molecules with a suitable energy level have usually been considered as photosensitizers rather than catalysts for photocatalytic hydrogen evolution (PHE). Herein, we achieved direct PHE using hydrate zinc tetraphenylporphyrin $\left(\mathrm{ZnP}, \mathrm{ZnTPP} \cdot \mathrm{H}_{2} \mathrm{O}\right)$ nanostructures synthesized by a liquid-phase chemical reaction as photocatalysts. The shape-dependent photocatalysis revealed that the $Z n P$ nanosheets (ZnP-NS) exhibit higher PHE activity $\left(\sim 0.16 \mathrm{mmol} \mathrm{g}^{-1} \mathrm{~h}^{-1}\right)$ than the $\mathrm{ZnP}$ octahedron nanoparticles (ZnP-NPs) ( $\left.0.06 \mathrm{mmol} \mathrm{g}^{-1} \mathrm{~h}^{-1}\right)$. After in situ construction of the rubrene/ZnP-NS heterostructure, more efficient PHE of this pure organic nanostructure was obtained due to the occurrence of photoinduced electron transfer and Förster resonance energy transfer (FRET). The optimal $\mathrm{PHE}$ rate is $\sim 0.56 \mathrm{mmol} \mathrm{g}^{-1} \mathrm{~h}^{-1}$. Furthermore, with the addition of $3.0 \mathrm{mM}$ methyl viologen (MV) and 3.8 wt\% platinum, a PHE rate of $\sim 9.3 \mathrm{mmol} \mathrm{g}^{-1} \mathrm{~h}^{-1}$ can be achieved at $\mathrm{pH}=7$. This study offers a new route to design organic small molecules as photocatalysts.
\end{abstract}

\section{Introduction}

Over the past half century, PHE from water splitting has attracted more and more attention because of the increasing worldwide energy shortage and environmental issues. ${ }^{1-7}$ With the rapid development of nanoscience and nanotechnology, obvious progress has been made in the photocatalysis of nanosemiconductors due to their abundant active sites and efficient absorption of solar light. ${ }^{1-5}$ To date, a variety of inorganic nanomaterials have been synthesized as photocatalysts such as transition metal oxides and metal sulfides. ${ }^{3-5}$ Although significant development has been achieved in the field of inorganic systems, there are few reports on PHE when organic small molecules nanostructures were used directly as photocatalysts rather than photosensitizers over the past two decades. ${ }^{6,7}$

In recent years, $\pi$-conjugated organic semiconductors, particularly organic dye molecules with high light-harvesting properties, have attracted considerable attention as a result of their relatively low-cost and various stable modifiable structures. ${ }^{8-10}$ To take metal porphyrin (MP) for example, theoretically, the porphyrin macrocycle can not only offer

${ }^{a}$ Key Laboratory of Design and Assembly of Functional Nanostructures, Chinese Academy of Sciences, Fuzhou, 350002, P. R. China

${ }^{b}$ Fujian Provincial Key Laboratory of Nanomaterials, Fujian Institute of Research on the Structure of Matter, Chinese Academy of Sciences, Fuzhou, Fujian 350002, P. R. China. E-mail: longtiank@firsm.ac.cn

'Beijing National Laboratory for Molecular Sciences (BNLMS), Institute of Chemistry, Chinese Academy of Sciences, Beijing 100190, P. R. China. E-mail: jnyao@iccas.ac.cn $\dagger$ Electronic supplementary information (ESI) available. See DOI: $10.1039 /$ c6ta01582d a unique platform for the coordination of different metal atoms to tune the spectroscopic and/or electrochemical properties of the chromophore, but also stabilize the redox-active metal centre. These advantages indicate their potential applications in photoelectric transfer. In fact, compared to the normal hydrogen electrode (NHE), the reduction potential of many MPs from $\mathrm{MP}^{+}$to MP excited stage (MP*), such as MgTPP, ZnTPP and AlTPP, is more negative and more positive than that from MP* to $\mathrm{MP}^{-}{ }^{11}$ This means that these MPs may give photogenerated electron to reduce a proton. With the help of an appropriate sacrificial electron donor (SED), PHE may be achieved via the application of a Z-scheme. ${ }^{7,9,12,13}$ Although the porphyrin-based PHE has been widely studied, to the best of our knowledge, there are few reports on directly splitting water for PHE using MPs as photocatalysts rather than photosensitizers., ${ }^{9,14}$ Now, more efforts are seemingly focused on constructing a molecule system for PHE than nanostructures.

The effect of organic nanostructures with different shapes, size, crystal types and self-assembly on the optoelectronic properties have been studied widely. ${ }^{15-20}$ In particular, the recent study of the self-assembly of naturally-occurring molecules provides a new opportunity to investigate and simulate the function of biological systems. For example, Yan's group successfully adjusted the optical properties of porphyrin based on peptide-tuning self-assembly method. ${ }^{18-20}$ This inspired us to explore the role of self-assembling porphyrin-based nanostructures analogous to natural photocatalytic system. At present, few reports on the PHE properties of organic small molecule nanostructures can be attributed to three factors. The first is the difficult production of large-scale monodisperse 
organic nanostrucutures. ${ }^{21}$ The second is their hydrophobic essence of main body, which means difficult diffusion of water or protons to the reaction interface and high overpotential. ${ }^{10}$ The third is the higher Coulomb binding energy of Frenkel exciton existed in an organic system than Wannier exciton in inorganic semiconductors, which prevents photoinduced electrons and holes from effectively separating., ${ }^{\mathbf{2 , 6 2 2}}$ To solve these tough problems, an entirely new strategy must be proposed. In this regard, we wondered whether these difficulties can be overcome by preparing hydrate organic nanostructures via a liquid-phase chemical reaction. ${ }^{16,23}$ In this way, a variety of monodisperse $\mathrm{ZnP}$ nanostructures can be synthesized on a large scale. It is very exciting that ZnP-NS with fully exposed crystal water on the crystal face can also be synthesized. ${ }^{23}$ Due to the existence of a hydrogen bond between ZnP and body-phase water via crystal water, in theory, ZnP-NS not only have well hydrophilicity, but also contribute to the transfer of a photoinduced electron from the metal atom on the porphyrin ring to proton $/ \mathrm{H}_{2} \mathrm{O} .{ }^{24}$ As far as the third problem was concerned, it was proved that the construction of a heterostructure is an effective route. ${ }^{25,26}$

Compared to hydrogen, $\mathrm{ZnP}$ has a more negative reduction potential from $\mathrm{ZnP}^{+}$to $\mathrm{ZnP}^{*}$ (whether single or triplet excited state) as well as a long triplet state lifetime, which implies that it may be a potential photocatalyst..11,12,27 For organic semiconductor rubrene, its far long diffusion distance of triplet excitons, more positive LUMO $(-2.10 \mathrm{eV})$ than $\mathrm{ZnP}(-2.39 \mathrm{eV})$ and excellent optical properties make it suitable as a photosensitizer when recombined with $\mathrm{ZnP} .{ }^{27-30}$ In this study, large amounts of ZnP-NS were synthesized via a liquid-phase chemical reaction and achieved direct PHE from water splitting when ZnP-NS and triethanolamine (TEOA) were used as the photocatalyst and sacrificial electron donor (SED), respectively. Through the in situ construction of rubrene/ZnP-NS heterostructure in the aqueous phase with ZnP-NS as templates, the efficiency of PHE was further improved. Moreover, it was also found that more efficient PHE can be observed when the system contains methyl viologen (MV) and $\mathrm{H}_{2} \mathrm{PtCl}_{6} \cdot 6 \mathrm{H}_{2} \mathrm{O}$.

\section{Results and discussions}

Fig. 1A shows the synthesis process of $\mathrm{ZnP}-\mathrm{NS}$ and rubrene/ZnP-NS nanostructures. In a typical experiment, $1 \mathrm{~mL}$ of $1 \mathrm{mM} \mathrm{ZnP}$ perchlorate $\left(10^{-6} \mathrm{~mol}\right)$ in acetonitrile was injected rapidly into $5 \mathrm{~mL}$ of an aqueous solution, including $0.5 \mathrm{M}$ poly(vinyl pyrrolidone) (PVP) and $10 \mathrm{mM}$ sodium nitrite $\left(\mathrm{NaNO}_{2}\right)$, at room temperature. ${ }^{23}$ As shown in Fig. $1 \mathrm{~B}$ by the scanning electron microscopy (SEM) images, the square nanoflakes with an edge length of $\sim 900 \mathrm{~nm}$ and a thickness of $<100 \mathrm{~nm}$ can be well synthesized. After centrifuging and washing, ZnP-NS were redispersed in ultrapure water to form a $1.0 \mathrm{mg} / 30 \mathrm{~mL}$ colloid dispersion system after mild sonication. To fabricate the rubrene/ZnP-NS heterostructure, the precursor of rubrene perchlorate was first synthesized according to the reported method. ${ }^{31}$ After it was added to water, MALDI-TOF mass spectroscopy in Fig. S1† proved the formation of the rubrene NPs, as shown in Fig. S2. $\uparrow$ Similarly, to obtain the composites of rubrene/ZnP with a molar ratio of $1: 6$, $1: 3$ and $2: 3,1 \mathrm{~mL}$ of $1 \mathrm{mM}$ rubrene perchlorate in acetonitrile was, respectively, added to $120 \mathrm{~mL}, 60 \mathrm{~mL}$ and $30 \mathrm{~mL}$ of a $1.0 \mathrm{mg} / 30 \mathrm{~mL}$ ZnP-NS aqueous dispersion and aged for 12 hours.
A

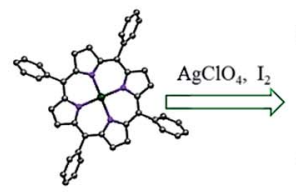

ZnTPP

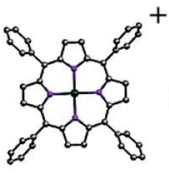

$\operatorname{ZnTPP}+$

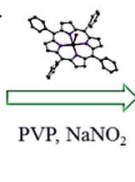

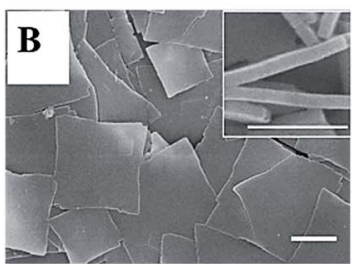
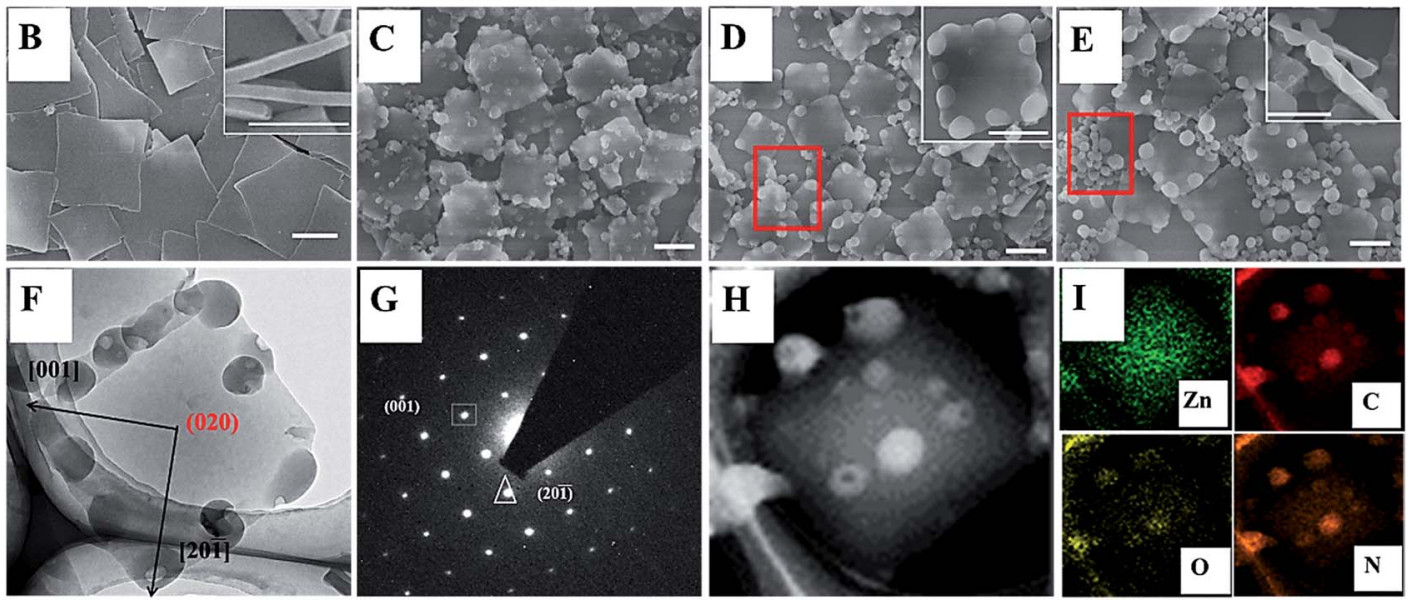

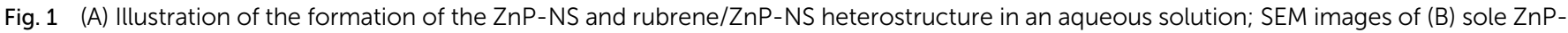

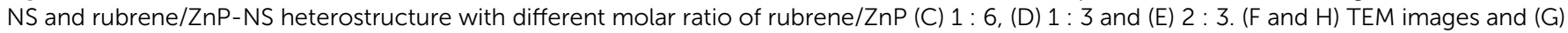

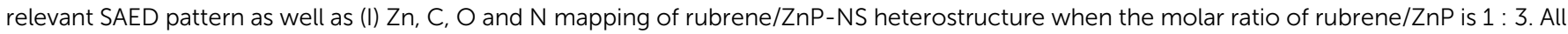
scale bars are $500 \mathrm{~nm}$. 
The as-prepared samples were characterized by SEM, transmission electron microscopy (TEM), selected area and elemental mapping. Fig. 1C-E shows that with increasing molar ratio of rubrene/ZnP from $1: 6,1: 3$ to $2: 3$, the electron diffraction (SAED) average size of rubrene NPs increases from $\sim 100 \mathrm{~nm}, \sim 150 \mathrm{~nm}$ to $\sim 200 \mathrm{~nm}$ and more detached rubrene NPs can be found, as marked by the red frames. This should result from the increase in rubrene monomer chemical potential after a rapid reaction with the decreasing volume of dispersion agent. ${ }^{23}$ Interestingly, Fig. $1 \mathrm{~F}$ and inset maps of Fig. 1D and E show that the rubrene NPs prefer to form at the edge of two smooth faces of ZnP-NS. This was attributed to the existence of rough faces on the edge of the ZnP-NS and higher reduction potential of rubrene ${ }^{+} /$rubrene $(0.9 \mathrm{eV}$ vs. SCE) than $\mathrm{ZnP}^{+} / \mathrm{ZnP}(0.71 \mathrm{eV}$ vs. SCE), which means not only the reaction between the rubrene cation and $\mathrm{ZnP}$, but also the preferred nucleation of rubrene at these rough faces with high surface energy. ${ }^{11,31}$ In addition, TEM image in Fig. 1F shows the close contact between the rubrene NPs and ZnP-NS, indicating the in situ formation of rubrene NPs on the surface of the ZnP-NS. The corresponding ED pattern in Fig. $1 \mathrm{G}$ confirms that a smooth crystal face of ZnP-NS can be indexed to (020) with fully exposed crystal water (see Fig. 1A). The nanosheet in Fig. $1 \mathrm{H}$ and relevant $\mathrm{Zn}$ element mapping in Fig. 1I further verify the synthesis of a high-quality rubrene/ZnP-NS nano-heterostructure and the morphology of $\mathrm{ZnP}$ remains unchanged after the formation of rubrene via chemical reaction.

To gain insight into the interaction between the ZnP-NS and rubrene, the optical properties of $\mathrm{ZnP}-\mathrm{NS}$, rubrene NPs and the composite of rubrene/ZnP-NS in the aqueous phase were studied via an analysis of their UV-vis absorption and steadystate photoluminescent (PL) spectra as well as time-resolved fluorescent lifetime (TRFL), as illustrated in Fig. 2. In Fig. 2A, the peak at $305 \mathrm{~nm}$ of the pristine rubrene NPs originates from $\mathrm{n}-\pi^{*}$ transitions, whereas the shoulder peak at $432 \mathrm{~nm}$, the two peaks at 450 and $515 \mathrm{~nm}$ as well as the two peaks in the range of 600-800 nm of ZnP-NS belong to the Soret-band, J-aggregate and Q-band from $\mathrm{a}_{1 \mu}(\pi)$ and $\mathrm{a}_{2 \mu}(\pi)$ to $\mathrm{e}_{\mathrm{g}}\left(\pi^{*}\right)$ electron transition, respectively. ${ }^{32}$ With increasing rubrene content, the UV-vis spectra $(\mathrm{a}-\mathrm{c})$ of the composites present two obvious phenomena compared to pristine ZnP-NS (d) and rubrene NPs (f). One is the enhancement of both rubrene absorption peaks and ZnP (J-aggregate and Q-band) absorption peaks, another is the redshift of rubrene and ZnP Q-band absorption peaks. It can be found that the absorption of rubrene NPs is shifted from $307 \mathrm{~nm}$ to $309 \mathrm{~nm}$, whereas the $\mathrm{ZnP}$ Q-band is shifted from $558 \mathrm{~nm}$ to $563 \mathrm{~nm}$ when the ZnP Soret-band remains constant. These phenomena strongly suggest a $\pi-\pi$ interaction between $\mathrm{ZnP}$ and rubrene in the composite, which in turn decreases the free energy of surface molecules of $\mathrm{ZnP}$ and rubrene and thus promotes the formation of more aggregates and intermolecular charge transfer. ${ }^{33}$

To further study the interaction between ZnP-NS and rubrene in the heterostructure, the fluorescence of the as-prepared samples was carried out, as shown in Fig. 2B. The figure shows that the PL spectrum of the rubrene NPs has an emission peak at $569 \mathrm{~nm}$ upon excitation at $350 \mathrm{~nm}$. However, the emission peak intensity of rubrene NPs decreases drastically and is red shifted to $577 \mathrm{~nm}$ after rubrene NPs formed on the surface of the ZnP-NS, which indicates the formation of a new excited state in the composites such as (rubrene-rubrene)* exciplex and (rubrene-ZnP)* excimer. $^{34}$ In this case, the fluorescence of rubrene NPs (c) at $\sim 570 \mathrm{~nm}$ can be quenched totally by a factor

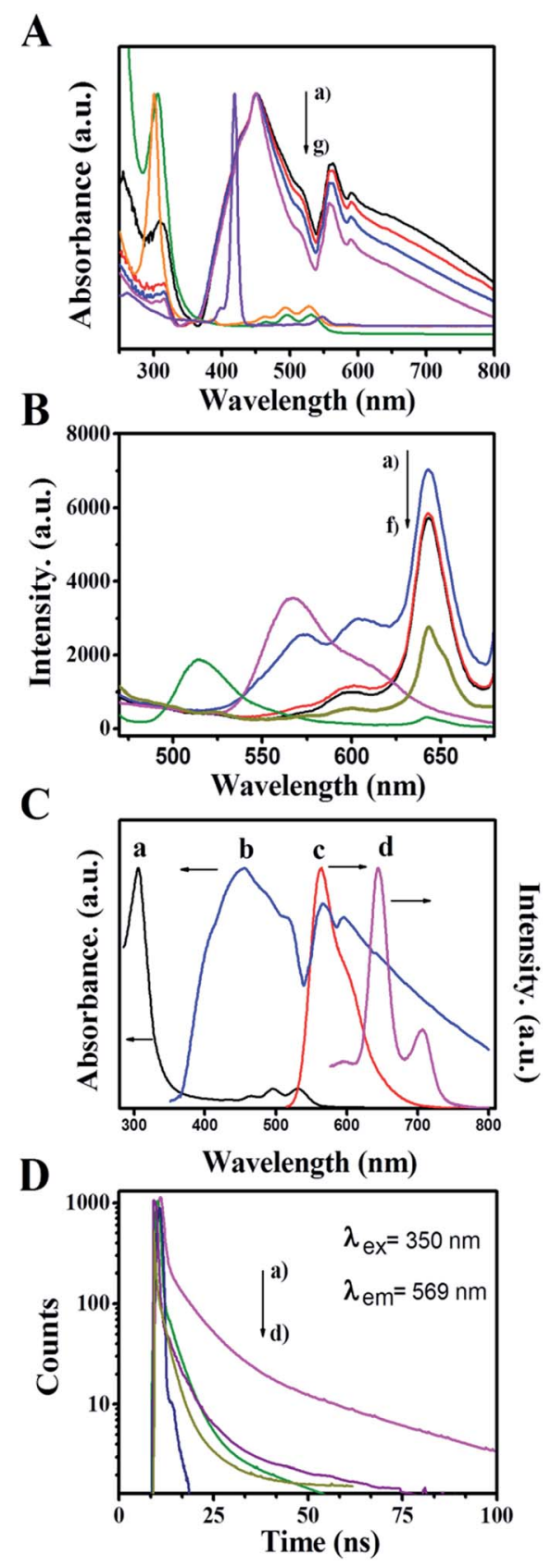

Fig. 2 In an aqueous dispersion, (A) UV-vis absorption spectra of rubrene/ZnP-NS NPs with molar ratio of rubrene/ZnP (a) $2: 3$, (b) $1: 3$ and (c) $1: 6$, (d) sole ZnP-NS and (f) rubrene NPs as well as (e) rubrene and (g) $\mathrm{ZnP}$ solution in $\mathrm{CH}_{2} \mathrm{Cl}_{2}$; (B) PL spectra of rubrene/ZnP-NS with molar of (a) $2: 3$, (b) $1: 3$ and (c) $1: 6$, (d) sole ZnP-NS and (e) rubrene NPs as well as (f) rubrene/ZnP-NS + MV; (C) the UV-vis absorption and $\mathrm{PL}$ spectra of ( $\mathrm{a}$ and $\mathrm{c}$ ) rubrene NPs and ( $\mathrm{b}$ and $\mathrm{d}$ ) ZnP-NS in aqueous dispersion. (D) Fluorescence decay profiles of (a) sole rubrene NPs and rubrene in rubrene/ZnP-NS composite with molar of (b) $2: 3$, (c) $1: 3$ and (d) $1: 6$. The nanodispersed system was monitored at $569 \mathrm{~nm}$ and the excitation wavelength was $350 \mathrm{~nm}$. 
of ten compared to the pristine rubrene NPs (e). The sudden increase of rubrene fluorescence (a) in Fig. 2B when the molar ratio of rubrene/ZnP is $2: 3$ should result from the existence of sole rubrene NPs, as shown in Fig. 1E. In addition, Fig. 2B displays that ZnP-NS has an emission peak at $643 \mathrm{~nm}$ upon excitation at $350 \mathrm{~nm}$. It can be found that the peak intensity obviously increases in the composites and the emission wavelength remains constant. Fig. 2C shows that the UV-vis absorption spectrum of ZnP Q-band (c) overlaps very well with the steady-state emission spectrum of the rubrene NPs (b) in the wavelength range of $600-700 \mathrm{~nm}$. This is essential for the FRET from rubrene to ZnP-NS. The existence of a $\pi-\pi$ interaction between $\mathrm{ZnP}$ and rubrene at the heterojunction also strongly indicates that the relative orientation of transition dipoles for the rubrene donor to the $\mathrm{ZnP}$ acceptor is coincident and the distance between rubrene and $\mathrm{ZnP}$ molecules is close, ${ }^{35}$ which meets requirements for the occurrence of FRET. Thus, FRET from rubrene to $\mathrm{ZnP}$ is responsible for the enhanced emission of $\mathrm{ZnP}$ in the heterostructure and indicates the formation of the excimer of (rubrene $\cdot \mathrm{ZnP}) *$ at the heterojunction.

The fluorescence decays of rubrene at $569 \mathrm{~nm}$ were measured using an excitation wavelength of $350 \mathrm{~nm}$, as shown in Fig. 2D. The average lifetime of rubrene NPs (a) in the aqueous phase is $12.5 \mathrm{~ns}$. In the case of rubrene/ZnP-NS heterostructure, the TRFLs of rubrene $(b-d)$ obviously become shorter as the content of rubrene decreased. As listed in Table 1, the decay curves of rubrene in all samples can be well fitted by three exponents, which means three relaxation processes of rubrene excited state $\left(\mathrm{S}^{*}\right)$. According to the corresponding excitation spectra (see Fig. S3†), it might be related to the transition from $\mathrm{S}^{*}$ to the exciplex or excimer $\left(\mathrm{S}_{\mathrm{e}}^{*}\right)$, aggregate $\left(\mathrm{S}_{\mathrm{a}}^{*}\right)$ and ground state $\left(\mathrm{S}_{0}\right)$. The longest TRFL $\left(\tau_{3}\right)$ should arise from $S^{*} \rightarrow S_{0}$, as reported earlier. ${ }^{36}$ For rubrene, the formation of heterojunction means the appearance of more excimers due to $\pi-\pi$ interaction between $\mathrm{ZnP}$ and rubrene. When the molar ratio of rubrene/ZnP is $>1: 3$ in the composite, there are more excimers with the increased content of rubrene. Based on this, $\tau_{1}$ should be from the transition of $\mathrm{S}^{*} \rightarrow \mathrm{S}_{\mathrm{e}}^{*}$ as referred to the percentage change of TRFL in Table 1 . Therefore, $\tau_{2}$ represents the lifetime of $\mathrm{S}^{*} \rightarrow \mathrm{S}_{\mathrm{a}}^{*}$. The corresponding TRFLs are $\tau\left(\mathrm{S}^{*} \rightarrow\right.$ $\left.\mathrm{S}_{0}\right)>\tau\left(\mathrm{S}^{*} \rightarrow \mathrm{S}_{\mathrm{a}}^{*}\right)>\tau\left(\mathrm{S}^{*} \rightarrow \mathrm{S}_{\mathrm{e}}^{*}\right)$. The data in Table 1 show that the TRFLs of $\tau_{1}$ and $\tau_{2}$ become longer as the content of rubrene increased. This may involve the size-effect of rubrene NPs.

Table 1 Fluorescence decay time of rubrene in pristine NPs and rubrene/ZnP-NS composites at $569 \mathrm{~nm}$. The excitation wavelength is $350 \mathrm{~nm}^{a}$

\begin{tabular}{|c|c|c|c|c|c|c|c|}
\hline \multirow{2}{*}{$\begin{array}{l}\text { Molar ratio } \\
\text { of rubrene/ZnP }\end{array}$} & \multicolumn{2}{|l|}{$\tau_{1}$} & \multicolumn{2}{|l|}{$\tau_{2}$} & \multicolumn{2}{|l|}{$\tau_{3}$} & \multirow[b]{2}{*}{$\tau^{\mathrm{a}} / \mathrm{ns}$} \\
\hline & ns & $\%_{1}$ & ns & $\%_{2}$ & ns & $\%_{3}$ & \\
\hline $1: 0$ & 0.55 & 37.9 & 6.96 & 36.4 & 37.86 & 25.7 & 12.47 \\
\hline $2: 3$ & 0.33 & 76.7 & 4.98 & 15.1 & 40.43 & 8.3 & 4.36 \\
\hline $1: 3$ & 0.32 & 69.5 & 3.69 & 22.1 & 36.78 & 8.4 & 4.13 \\
\hline $1: 6$ & 0.28 & 64.0 & 2.30 & 29.9 & 32.13 & 6.1 & 2.83 \\
\hline
\end{tabular}

Smaller size means higher energy level of $\mathrm{S}_{\mathrm{e}}^{*}$ and/or $\mathrm{S}_{\mathrm{a}}^{*}$. In a word, compared to pristine rubrene NPs, the formation of more rubrene-related excimers and exciplex at the heterojunction is responsible for the quenching of rubrene PL and the enhancement of ZnP PL. Under the excitation of light, it will result in the appearance of more photoinduced electrons on the surface of ZnP-NS and achieve more efficient utilization of light. ${ }^{35}$

To further investigate the effect of the formation of rubrene/ ZnP-NS heterojunction on the photoelectric properties, PHE activity of different samples was studied when they were used as photocatalysts with TEOA as SED. PHE was carried out under a $300 \mathrm{~W}$ Xe lamp after $2 \mathrm{mg}$ of sample was dispersed in $90 \mathrm{~mL}$ ultrapure water by sonication and $10 \mathrm{~mL}$ of TEOA was added to it. To survey the shape-dependent photocatalysis of the ZnPs, ZnP-NPs with an average size of $\sim 80 \mathrm{~nm}$ were synthesized (see Fig. $\mathrm{S} 4 \dagger$ ). Fig. 3A shows the time course of PHE over the ZnP-NPs, ZnP-NS and rubrene/ZnP-NS heterostructures. The ZnP-NS presents higher photocatalytic activity than ZnP-NPs. The PHE rate of ZnP-NS is $\sim 0.16 \mathrm{mmol} \mathrm{g}^{-1} \mathrm{~h}^{-1}$, whereas that of ZnP-NPs is $\sim 0.06 \mathrm{mmol} \mathrm{g}^{-1} \mathrm{~h}^{-1}$. This indicates that the catalytic activity of the $\mathrm{ZnP}$ nanostructure may be closely associated with its shape. Combined with the crystal structure of ZnP-NS in Fig. 1A, it is believed that the active sites of $\mathrm{ZnP}$ were concentrated on the (020) crystal face. Compared to pristine ZnP-NS and rubrene NPs, the heterostructures of rubrene/ZnP-NS constructed on the $\pm(020)$ crystal faces of ZnP-NS reveal more efficient PHE activity.

The experimental results in Fig. 3A show that the loading of rubrene plays an important role in adjusting the photocatalytic activity of the heterostructure. With increasing content of rubrene, the amount of hydrogen first increases and then decreases when the molar ratio of rubrene/ZnP is changed from $1: 6,1: 3$ to $2: 3$. As we know, the photocatalytic activity of a nanocomposite is closely related to two factors. One is the ability to harvest light, another is the amount of photocatalytic active sites at the surface. The increase in heterojunction usually means a decrease in the number of active sites. In our system, the PL spectra in Fig. 2A clearly display that the intensity of UV-vis absorption enhances as the content of rubrene increased. However, the amount of heterojunction, in fact, did not increase when the molar ratio of rubrene/ZnP was increased to $2: 3$. As observed in Fig. 1E, there are a large number of independent rubrene NPs, which have little photocatalytic activity in our experiment. In nature, the PL spectra of curves (b) and (c) in Fig. 2B also imply that increasing the loading of rubrene to ZnP-NS will not improve the energy and/or electron transfer from rubrene to $\mathrm{ZnP}$. The optimal molar ratio of rubrene/ZnP for PHE is $1: 3$. Its PHE rate is $0.56 \mathrm{mmol} \mathrm{g}^{-1} \mathrm{~h}^{-1}$ and it is more than three times than that of pure ZnP-NS, which confirms that the formation of the rubrene/ZnP-NS heterojunction has a positive effect on the PHE from water splitting. Actually, the result is better than the PHE rate of ZnP-NS with $\sim 3.8$ wt $\%$ Pt loaded, as illustrated in Fig. S5. $\dagger$ In this system, the turnover numbers $\left(\mathrm{TON}_{\mathrm{ZnP}}\right)$ and the turnover frequency $\left(\mathrm{TOF}_{\mathrm{ZnP}}\right)$ for the $\mathrm{ZnP}$ are $\sim 5$ and $2.72 \times 10^{-4} \mathrm{~s}^{-1}$ after 5 hours irradiation, respectively. Furthermore, the PHE durability of the 


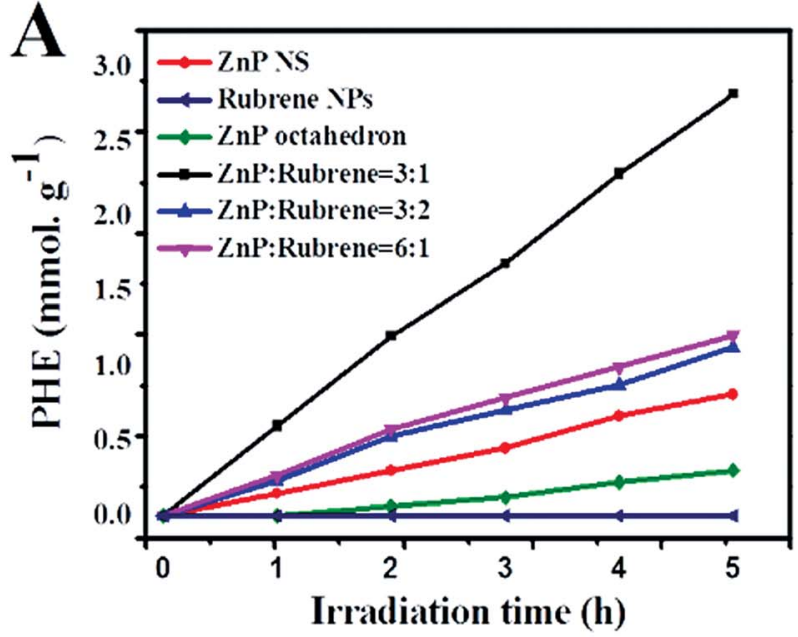

B

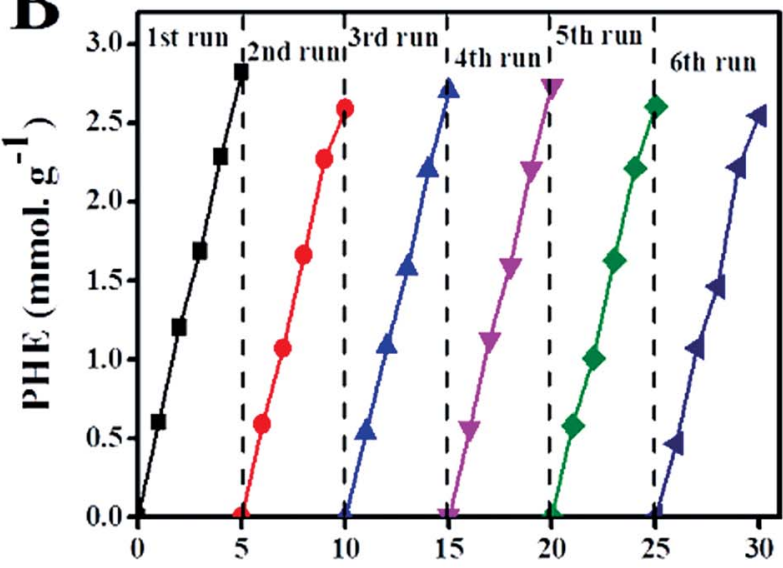

Irradiation time (h)

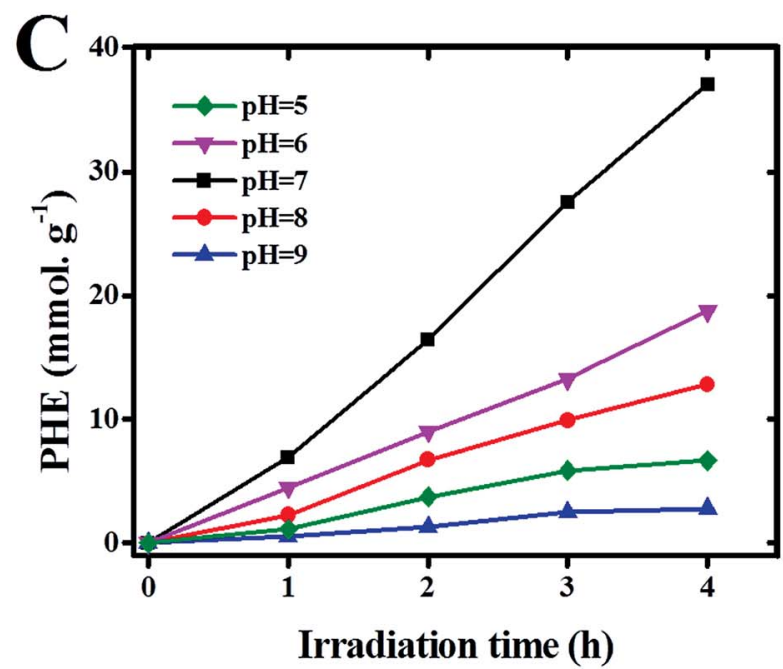

Fig. 3 (A) Time course of PHE of ZnP-NPs, ZnP-NS, rubrene NPs and rubrene/ZnP-NS heterostructure with different molar ratios $(6: 1,3: 1$ and $3: 2$ ); (B) stability test of PHE (every $5 \mathrm{~h}$ ) and (C) time course of PHE of rubrene/ZnP-NS $(3: 1)$ at different $\mathrm{pH}$ in the presence of $3.0 \mathrm{mM}$ $\mathrm{MV}, 10 \mathrm{~mL}$ TEOA and $3.8 \mathrm{wt} \% \mathrm{Pt}$ in $100 \mathrm{~mL}$ aqueous system.

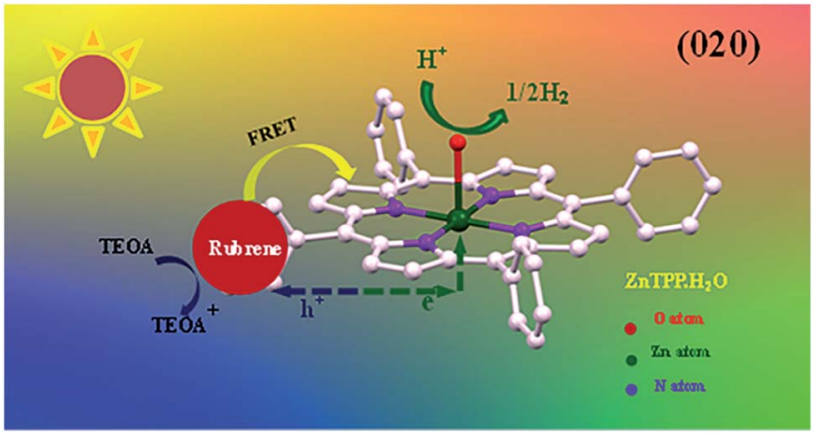

Scheme 1 Schematic photoexcited electron transfer and FRET as well as $\mathrm{PHE}$ over the rubrene/ZnP-NS photocatalyst under light irradiation.

rubrene/ZnP-NS $(1: 3)$ heterostructure was performed. The results can be seen in Fig. 3B. For each cycle, there is no evident decay of PHE activity, indicating the excellent photocatalytic stability of the composite.

As a matter of fact, zinc porphyrin and its derivatives are usually used for PHE in a three component system, including MV and Pt. ${ }^{14}$ As shown in Fig. 2B, the PL spectrum (f) of the heterostructure at $643 \mathrm{~nm}$ was quenched by a factor of nineteen after the addition of MV. In view of this, we tried constructing the system of TEOA/rubrene/ZnP-NS/MV/Pt. The results are displayed in Fig. 3C. Taking the effect of the pH on PHE into account, we achieved a PHE rate of $\sim 9.3 \mathrm{mmol} \mathrm{g}^{-1} \mathrm{~h}^{-1}$ at $\mathrm{pH}=$ 7. After 4 hours irradiation, the $\mathrm{TON}_{\mathrm{Pt}}$ and $\mathrm{TOF}_{\mathrm{Pt}}$ were 385 and $2.68 \times 10^{-2} \mathrm{~s}^{-1}$, respectively. The TON $\mathrm{ZnP}$ was $\sim 65$ and the $\mathrm{TOF}_{\mathrm{ZnP}}$ was $4.51 \times 10^{-3} \mathrm{~s}^{-1}$. In addition, at a wavelength of 365 $\pm 10 \mathrm{~nm}$, a quantum yield (Ø) of $1.65 \%$ was obtained. In this type of system, MV is usually used as an electron relay to transfer photoinduced electrons from a photosensitizer to the Pt surface, whereas Pt is often seen as a catalyst. ${ }^{9}$ The existence of Pt further improves the efficiency of photocatalytic hydrogen evolution (PHE) by limiting the recombination of photoinduced electrons and holes due to the Schottky effect and providing more and better activity sites for the reaction from the proton to hydrogen and the desorption of hydrogen. In the photocatalytic system of TEOA/rubrene/ZnP-NS/MV/Pt, the effect of $\mathrm{pH}$ on the PHE results from two factors. (1) A higher $\mathrm{pH}$ value will decrease the concentration of protons in water and in turn reduce the reaction rate from protons to hydrogen. (2) When hydrochloric acid $(\mathrm{HCl})$ was used to adjust the $\mathrm{pH}$, a lower $\mathrm{pH}$ value may influence the activity of TEOA as a sacrificial electron donor. Therefore, there is an optimal pH in our experiment.

Based on our experiments, we established the schematic of PHE of rubrene/ZnP-NS, as illustrated in Scheme 1. Under solar light illumination, both $\mathrm{ZnP}$ and rubrene are pumped to the excited state due to an electron transition and then form excitons (the pair of electron and hole), as shown in eqn (1) and (2).

$$
\begin{gathered}
\text { Rubrene } \stackrel{h v}{\longrightarrow} \text { rubrene }^{*} \leftrightarrow \text { rubrene }^{+}+\mathrm{e} \\
\mathrm{ZnP} \stackrel{h v}{\longrightarrow} \mathrm{ZnP}^{*} \leftrightarrow \mathrm{ZnP}^{+}+\mathrm{e}
\end{gathered}
$$


In addition, FRET between the excited-state rubrene and ground-state $\mathrm{ZnP}$ occurs and generates an excimer, finally forms ground-state rubrene and excited-state $\mathrm{ZnP}$ because of the $\pi-\pi$ stacking interaction and close contact between $\mathrm{ZnP}$ and rubrene at the heterojunction, as referred in eqn (3). This process promotes the occurrence of reaction (2).

$$
\begin{aligned}
\text { Rubrene* }+\mathrm{ZnP} & \rightarrow(\text { rubrene } \cdot \mathrm{ZnP})^{*} \\
& \rightarrow \text { rubrene }+\mathrm{ZnP}^{*}
\end{aligned}
$$

Because the LUMO and HOMO of $\mathrm{ZnP}$ and rubrene are -2.39 $\mathrm{eV}$ and $-5.13 \mathrm{eV}$ and $-2.10 \mathrm{eV}$ and $-4.62 \mathrm{eV}$, respectively, higher energy photogenerated electrons of rubrene should transfer to ZnP, and higher energy photogenerated holes of ZnP should transfer to rubrene, as shown in eqn (4) and (5). ${ }^{27,30}$ In this process, the TRFLs of both rubrene and ZnP are obviously lowered (see Tables 1, S1 and Fig. S6†).

$$
\begin{aligned}
& \text { Rubrene }^{-}+\mathrm{ZnP} \rightarrow \text { rubrene }+\mathrm{ZnP}^{-} \\
& \text {Rubrene }+\mathrm{ZnP}^{+} \rightarrow \text { rubrene }^{+}+\mathrm{ZnP}
\end{aligned}
$$

On the (020) crystal face of ZnP-NS, the photoinduced electrons from $\mathrm{ZnP}$ and rubrene first transfer from ligand TPP to the central atom of $\mathrm{Zn}(\mathrm{II})$, then to crystal water, finally to water and/or protons in body phase. ${ }^{24}$ Subsequently, as listed in eqn (6) and (7) and Scheme 1, holes on the surface of rubrene were reduced by TEOA and hydrogen is generated at the active sites.

$$
\begin{gathered}
\text { Rubrene }^{+}+\text {TEOA } \rightarrow \text { rubrene }+ \text { TEOA }^{+} \\
\mathrm{H}^{+}+\mathrm{e} \rightarrow \mathrm{H}_{2}
\end{gathered}
$$

According to the abovementioned results and analysis for the photocatalytic system of TEOA/rubrene/ZnP-NS, we believe that in the entire process of PHE from water, (1) ZnP-NS is the photocatalyst, (2) the crystal water of the ZnP-NS (020) crystal face may not only play a bridge role in the transfer of photogenerated electron to protons, but also be a reaction site of proton to hydrogen, and (3) rubrene only acts as a photosensitizer.

\section{Conclusions}

In summary, we achieved direct PHE using the nanostructures of organic small molecule as photocatalysts. In this study, a series of nanostructures, including ZnP-NS, ZnP-NPs, rubrene NPs and heterostructures of rubrene/ZnP-NS with different molar ratios, were synthesized via a liquid-phase chemical reaction for PHE from water splitting. The shape-dependent PHE activity showed that ZnP-NS presented higher PHE activity than ZnP-NPs because of more exposed crystal water on the surface. The PHE rate of ZnP-NS was $\sim 0.16 \mathrm{mmol} \mathrm{g}^{-1} \mathrm{~h}^{-1}$. After in situ constructing the heterostructure of rubrene/ZnP-NS in an aqueous solution, FRET from rubrene to $\mathrm{ZnP}$ occurs, and in turn markedly promotes the capability of light absorption of $\mathrm{ZnP}$ and limits the recombination of photoinduced electrons and holes. The PHE rate of the optimal heterostructure was $\sim 0.56 \mathrm{mmol} \mathrm{g}^{-1} \mathrm{~h}^{-1}$. Moreover, the value of $\sim 9.3 \mathrm{mmol} \mathrm{g}^{-1} \mathrm{~h}^{-1}$ can be obtained at $\mathrm{pH}=7$ when the optimal composite of rubrene/ZnP-NS was added to the system including MV and colloid Pt. Our study not only demonstrates that the nanostructures of organic small molecules can be used directly as a photocatalyst, but also paves a new way for PHE from water splitting.

\section{Experimental}

\section{Materials}

Zinc tetraphenylporphyrin (ZnTPP) (95\%, J\&K Chemicals Co.), rubrene (98.5\%, J\&K Chemicals Co.), silver perchlorate (97\%, J\&K Chemicals Co.), iodine (99.5\%, Sinopharm Chemical Reagent Co.), anhydrous dichloromethane (J\&K Chemicals Co.) and acetonitrile (J\&K Chemicals Co., water $<30 \mathrm{ppm}$ ) were used in this study. Ultrapure water with a resistivity of $18.2 \mathrm{M} \Omega \mathrm{cm}^{-1}$ was produced using a Water Purifier apparatus (WP-UP-IV-20). Triethanolamine (99\%, Sinopharm Chemical Reagent Co.) and methyl viologen (98\%, J\&K Chemicals Co.) were used for photocatalysis. Hydrochloric acid ( $\mathrm{HCl}, 36-38 \%$, Sinopharm Chemical Reagent Co.) was used to adjust the pH. All chemicals were used without further purification.

\section{Preparation of ZnP-NS}

ZnP-NS were synthesized by a liquid phase chemical reaction method. First, the precursor of ZnTPP perchlorate was produced via reaction (8): ${ }^{23}$

$$
2 \mathrm{ZnTPP}+2 \mathrm{AgClO}_{4}+\mathrm{I}_{2} \rightarrow 2 \mathrm{ZnTPPP} \cdot \mathrm{ClO}_{4}+2 \mathrm{AgI} \downarrow
$$

In a typical synthesis, a solution of dry silver perchlorate in anhydrous acetonitrile $(2 \mathrm{~mL}, 100 \mathrm{mM})$ and a solution of $\mathrm{I}_{2}$ in anhydrous dichloromethane ( $4 \mathrm{~mL}, 25 \mathrm{mM}$ ) were added to a 10 $\mathrm{mL}$ solution of $20 \mathrm{mM}$ ZnTPP in anhydrous dichloromethane with stirring for 3 hours and aging for one hour. Silver chloride was filtered by centrifuging at $10000 \mathrm{rpm}$ for $10 \mathrm{~min}$. The solution of $\mathrm{ZnTPP} \cdot \mathrm{ClO}_{4}$ was then poured into $80 \mathrm{~mL}$ petroleum ether and sealed at room temperature for $3 \mathrm{~h}$. Finally, the green-black precipitate was obtained by centrifuging at $10000 \mathrm{rpm}$ for $10 \mathrm{~min}$ and further washed twice with petroleum ether. The as-obtained $\mathrm{ZnTPP} \cdot \mathrm{ClO}_{4}$ was dried under vacuum for further use. Second, $\mathrm{ZnTPP} \cdot \mathrm{ClO}_{4}(1 \mathrm{~mL}, 1 \mathrm{mM})$ in anhydrous acetonitrile was injected rapidly into $5 \mathrm{~mL}$ ultrapure water solution containing $10 \mathrm{mM}$ sodium nitrite and $500 \mathrm{mM}$ polyvinyl pyrrolidone (PVP). The nanosheets can be obtained according to reaction (9):

$$
\begin{aligned}
& 2 \mathrm{ZnTPP} \cdot \mathrm{ClO}_{4}+\mathrm{NaNO}_{2}+3 \mathrm{H}_{2} \mathrm{O} \rightarrow \\
& 2 \mathrm{ZnTPP} \cdot \mathrm{H}_{2} \mathrm{O}+\mathrm{NaNO}_{3}+2 \mathrm{HClO}_{4}
\end{aligned}
$$

After injection of the precursor and aging 3 hours, the colloidal suspension of ZnP-NS was found. The sample was separated and washed three times with pure water to remove the excess PVP. The final product was dried for further use. 


\section{Preparation of rubrene nanostructure}

First, the rubrene precursor was produced via reaction (10): ${ }^{31}$

$$
2 \mathrm{Rubrene}+2 \mathrm{AgClO}_{4}+\mathrm{I}_{2} \rightarrow 2 \text { rubrene } \cdot \mathrm{ClO}_{4}+2 \mathrm{AgI} \downarrow
$$

An olive-drab rubrene $\cdot \mathrm{ClO}_{4}$ solution was obtained. The solution was then treated by decompressed distillation to remove the solvent. The as-obtained rubrene $\cdot \mathrm{ClO}_{4}$ was dried under vacuum at room temperature. Second, rubrene $\cdot \mathrm{ClO}_{4}(1$ $\mathrm{mL}, 1 \mathrm{mM}$ ) in anhydrous acetonitrile was injected rapidly into a $3 \mathrm{~mL}$ pure water solution. After injection and aging for 3 hours, the rubrene nanostructure was obtained. The sample was then washed twice with pure water and dried for further use. Rubrene was prepared via reaction (11):

$$
\text { Rubrene }^{+} \stackrel{\mathrm{H}_{2} \mathrm{O}}{\longrightarrow} \text { Rubrene }
$$

\section{Characterization}

The morphologies and elemental composition were measured by a field emission scanning electron microscope (FESEM, Hitachi SU8010) and a transmission electron microscope (TEM, F20). The UV-vis absorption spectra were obtained using a UV-vis spectrophotometer (Shimadzu, UV2550). The photoluminescence (PL) spectra were acquired on a Hitachi F-4600 fluorescence spectrophotometer. The fluorescence lifetime decay spectra were performed with a transient state fluorescence spectrometer (Edinburgh Instrument, FLS980). The mass spectrum was measured using MALDI-TOF-MS spectrometer (Bruker, Autoflex III).

\section{Photocatalysis experiments}

The photocatalytic water splitting reactions were performed with a Pyrex top-irradiation reaction vessel connected to a closed glass gas circulation system (Labsolar III AG, Beijing Perfect-light Technology Co. Ltd). In a typical experiment, $2 \mathrm{mg}$ of ZnP-NS or rubrene/ZnP-NS (2 mg) was added to $90 \mathrm{~mL}$ ultrapure water, and $10 \mathrm{~mL}$ TEOA was then added. The irradiation light source was a $300 \mathrm{~W}$ Xe lamp (PLS-SXE 300). In the case of $\mathrm{H}_{2} \mathrm{PtCl}_{6} \cdot 6 \mathrm{H}_{2} \mathrm{O}\left(3.86 \times 10^{-7} \mathrm{~mol}\right)$ and $\mathrm{MV}^{2+}\left(2.95 \times 10^{-4}\right.$ $\mathrm{mol})$, after all regents were added to $100 \mathrm{~mL}$ reaction agents, the $300 \mathrm{~W}$ Xe lamp was applied for 2 hours so that $\mathrm{H}_{2} \mathrm{PtCl}_{6}$ could be reduced to $\mathrm{Pt}$ nanoparticles and to deposit on the surface of the ZnP-NS via reaction ${ }^{37}$ (12):

$$
\left[\mathrm{PtCl}_{6}\right]^{2-}+4 \mathrm{e}^{-} \rightarrow 6 \mathrm{Cl}^{-}+\mathrm{Pt}
$$

The $\mathrm{pH}$ was adjusted with $\mathrm{HCl}$. The evolved gas was analyzed by gas chromatography (GC 7900, Shanghai Techcomp Instrument Ltd). A flow of cooling water was used to maintain the reactant solution at room temperature.

The estimated turnover numbers (TON), turnover frequency (TOF) and quantum yield of hydrogen production $(\varnothing)$ were obtained using these equations:

$$
\mathrm{TON}=\frac{n_{\mathrm{H}_{2}}}{n_{\mathrm{Pt}}\left(\text { or } n_{\mathrm{ZnP}}\right)}
$$

$$
\begin{gathered}
\mathrm{TOF}=\frac{n_{\mathrm{H}_{2}}}{t \times n_{\mathrm{Pt}}\left(\text { or } n_{\mathrm{ZnP}}\right)} \\
\varnothing=\frac{2 n_{\mathrm{H}_{2}}}{I_{0} t}
\end{gathered}
$$

$n_{\mathrm{H}_{2}}$ represents the moles of hydrogen production. $n_{\mathrm{Pt}}$ is the moles of $\mathrm{Pt}$, whereas $n_{\mathrm{ZnP}}$ is the moles of $\mathrm{ZnPt}$ is the irradiation time. $I_{0}$ is the number of photons per second. The quantum efficiency $(\varnothing)$ was obtained by applying a Xe lamp $(300 \mathrm{~W})$ with a $365 \mathrm{~nm}$ bandpass filter.

\section{Acknowledgements}

The authors gratefully acknowledge the financial from the National Natural Science Foundation of China (No. 21252001, 21473204), the Special Project of National Major Scientific Equipment Development of China (No. 2012YQ120060), the Natural Science Foundation of Fujian Province (2015J01070), and the Science and Technology Planning Project of Fujian Province, Grant No. 2014H2008.

\section{Notes and references}

1 H. Tong, S. Ouyang, Y. Bi, N. Umezawa and M. Oshikiri, Adv. Mater., 2012, 24, 229-251.

2 F. X. Xiao, J. Miao, H. B. Tao, S. F. Hung, H. Y. Wang, H. B. Yang, J. Chen, R. Chen and B. Liu, Small, 2015, 11, 2115-2131.

3 I. Paramasivam, H. Jha, N. Liu and P. Schmuki, Small, 2012, 8, 3073-3103.

4 T. Y. Zhai, X. S. Fang, L. Li, Y. Bando and D. Golberg, Nanoscale, 2010, 2, 168-187.

5 Z. J. Han, F. Qiu, R. Eisenberg, P. L. Holland and T. D. Krauss, Science, 2012, 338, 1321-1324.

6 D. H. Wang, J. N. Pan, H. H. Li, L. J. Liu, Y. B. Wang and L. T. Kang, J. Mater. Chem. A, 2015, 4, 290-296.

7 Y. M. Tian, K. E. Martin, J. Y. Shelnutt, L. Evans, T. Busani, J. E. Miller, C. J. Medforth and J. A. Shelnutt, Chem. Commun., 2011, 47, 6069-6071.

8 A. Mishra and P. Bauerle, Angew. Chem., Int. Ed., 2012, 51, 2020-2067.

9 K. Ladomenou, M. Natali, E. Iengo, G. Charalampidis, F. Scandola and A. G. Coutsolelos, Coord. Chem. Rev., 2015, 304-305, 38-54.

10 J. L. Brédas, B. Beljonne, V. Coropceanu and J. Cornil, Chem. Rev., 2004, 104, 4971-5003.

11 J. P. Darwent, P. Douglas, A. Harriman, G. Porter and M. C. Richoux, Coord. Chem. Rev., 1982, 44, 83-126.

12 I. Okura and N. K. Thuan, J. Mol. Catal., 1979, 6, 227-230.

13 K. Maeda, ACS Catal., 2013, 3, 1486-1503.

14 A. J. Esswein and D. G. Nocera, Chem. Rev., 2007, 107, 40224047.

15 J. W. Lee, K. Kim, J. S. Jung, S. G. Jo, H. M. Kim, H. S. Lee, J. Y. Kim and J. S. Joo, Org. Electron., 2012, 13, 2047-2055. 
16 L. T. Kang, Z. C. Wang, Z. W. Cao, Y. Ma, H. B. Fu and J. N. Yao, J. Am. Chem. Soc., 2007, 129, 7305-7312.

17 L. W. Huang, Q. Liao, Q. Shi, H. B. Fu, J. S. Ma and J. N. Yao, J. Mater. Chem., 2010, 20, 159-166.

18 Q. L. Zou, L. Zhang, X. H. Yan, A. H. Wang, G. H. Ma, J. B. Li, H. Mohwald and S. Mann, Angew. Chem., Int. Ed., 2014, 53, 2366-2370.

19 K. Liu, R. R. Xing, C. J. Chen, G. Z. Shen, L. Y. Yan, Q. L. Zou, G. H. Ma, H. Mohwald and X. H. Yan, Angew. Chem., Int. Ed., 2015, 54, 500-505.

20 Q. L. Zou, K. Liu, M. Abbas and X. H. Yan, Adv. Mater., 2016, 28, 1031-1043.

21 H. Kasai, H. S. Nalwa, H. Oikawa, S. Okda, H. Matsuda, N. Minami, A. Kakuta, K. Ono, A. Mukoh and H. Nakanishi, Jpn. J. Appl. Phys., Part 2, 1992, 31, 1132-1134. 22 S. J. A. Moniz, S. A. Shevlin, D. J. Martin, Z. X. Guo and J. W. Tang, Energy Environ. Sci., 2015, 8, 731-759.

23 L. T. Kang, H. B. Fu, X. Q. Cao, Q. Shi and J. N. Yao, J. Am. Chem. Soc., 2011, 133, 1895-1901.

24 J. H. Yang, Y. J. Gao, W. Zhang, P. Tang, J. Tan, A. H. Lu and D. Ma, J. Phys. Chem. C, 2013, 117, 3785-3788.

25 Z. Zhao, Y. J. Sun and F. Dong, Nanoscale, 2015, 7, 15-37.

26 H. L. Wang, L. S. Zhang, Z. G. Chen, J. Q. Hu, S. J. Li, Z. H. Wang, J. S. Liu and X. C. Wang, Chem. Soc. Rev., 2014, 43, 5234-5244.
27 A. Irfan, N. Hina, A. G. Al-Sehemi and A. M. Asiri, J. Mol. Model., 2012, 18, 4199-4207.

28 H. Najafov, B. Lee, Q. Zhou, L. C. Feldman and V. Podzorov, Nat. Mater., 2010, 9, 938-943.

29 S. Tavazzi, A. Borghesi, A. Papagni, P. Spearman, L. Silvestri, A. Yassar, A. Camposeo, M. Polo and D. Pisignano, Phys. Rev. B: Condens. Matter Mater. Phys., 2007, 75(1-5), 245416.

30 F. Anger, R. Scholz, E. Adamski, K. Broch, A. Gerlach, Y. Sakamoto and T. Suzuki, Appl. Phys. Lett., 2013, 102(15), 013308.

31 L. Luo, G. Liu, L. W. Huang, X. Q. Cao, M. Liu, H. B. Fu and J. N. Yao, Appl. Phys. Lett., 2009, 95(1-3), 263312.

32 M. Nappa and J. S. Valentine, J. Am. Chem. Soc., 1977, 100, 5075-5080.

33 H. B. Fu and J. N. Yao, J. Am. Chem. Soc., 2001, 123, 14341439.

34 T. M. R. Viseu, G. Hungerford, A. F. Coelho and M. I. C. Ferreira, J. Phys. Chem. B, 2003, 107, 13300-13312.

35 S. Sardar, P. Kar, H. Remita, B. Liu, P. Lemmens, S. K. Pal and S. Ghosh, Sci. Rep., 2015, 5(1-14), 17313.

36 L. W. Huang, Q. Liao, Q. Shi, H. B. Fu, J. S. Ma and J. N. Yao, J. Mater. Chem., 2010, 20, 159-166.

37 S. Chen, Y. Qi, T. Hisatomi, Q. Ding, T. ASai, Z. Li, S. S. K. Ma, F. X. Zhang, K. Domen and C. Li, Angew. Chem., Int. Ed., 2015, 54, 8498-8501. 